\title{
Transcription analysis of chlorophyll biosynthesis in wildtype and chlorophyll b-lacking rice (Oryza sativa L.)
}

\author{
M.K. NGUYEN ${ }^{*, * * * * *, \#, ~ T . H . ~ S H I H *, ~ S . H . ~ L I N ", ~ W . D ~ H U A N G ~}{ }^{*}$, , and C.M. YANG ${ }^{*, \#,+}$ \\ Biodiversity Research Center, Academia Sinica, Nangang, Taipei 115, Taiwan* \\ Biodiversity Program, Taiwan International Graduate Program, Biodiversity Research Center, Academia Sinica and \\ National Taiwan Normal University, Taipei 115, Taiwan ${ }^{* *}$ \\ Department of Life Science, National Taiwan Normal University, Daan, Taipei 115, Taiwan ${ }^{* * *}$ \\ Faculty of Applied Sciences, Ton Duc Thang University, Ho Chi Minh, 70000, Vietnam ${ }^{\#}$ \\ Department of Agronomy, National Taiwan University, Daan, Taipei 106, Taiwan ${ }^{\# \#}$
}

\begin{abstract}
The aim of the present study was to investigate the photosynthetic properties and transcriptomic profiles of wildtype and chlorophyll (Chl) b-lacking rice (Oryza sativa L.). The plastid ultrastructure of the Chl b-lacking rice (i.e., loss of starch granules, abundant vesicles, and abundant plastoglobuli) indicated abnormal plastid development, whereas the analysis of transcriptome profiles and differentially expressed genes revealed that gene encoding PsbR (PSII core protein) was downregulated in the mutant, thereby reducing the Chl accumulation of the mutant. Meanwhile, in regards to Chl biosynthesis and degradation pathways, GluTR gene was downregulated, whereas UROD, CPOX, and $M g C H$ genes were upregulated. The qPCR results were generally consistent with those of the transcription analysis, except for the finding that NOL genes, which regulate $\mathrm{Chl} b$ degradation, were upregulated. These results suggest that both the reduction in Chl accumulation and increase in conversion rate of $\mathrm{Chl} b$ to $\mathrm{Chl} a$ caused $\mathrm{Chl} a / b$ ratio amplification in mutant. The present study also provides evidence for $\mathrm{Chl} b$ degradation via pheophorbide $b$.
\end{abstract}

Additional key words: grana; next-generation sequencing; photosynthesis; RNA-Seq; transcriptome.

\section{Introduction}

Photosynthesis (i.e., the process of converting sunlight energy into a chemically stable form), occurs in plants, algae, cyanobacteria, and other photosynthetic bacteria (Jin et al. 2003). In higher plants, photosynthesis occurs in chloroplasts, which are specific organelles that synthesize and degrade billion tons of chlorophyll (Chl) annually (Eckhardt et al. 2004, Liu et al. 2007, Kräutler 2008). Chl is extremely important during the photosynthesis, since it plays an essential role in light absorption and energy transfer (Fromme et al. 2003, Tanaka and Tanaka 2006). Therefore, understanding the efficiency of Chl biosynthesis could facilitate increases in photoassimilates accumulation, and ultimately, in crop yield (Mitchell and Sheehy 2006, Huang et al. 2013). Recently, the Chl biosynthesis and degradation pathways have been well characterized and found to involve more than 17 identified enzymes and 15 reactions from precursor 5-aminolaevulinic acid (ALA) to the $\mathrm{Chl} a$ and $\mathrm{Chl} b$ at $\mathrm{Chl}$ cycle until breakdown at a degradation pathway (Masuda and Fujita 2008). Even though Chl $a$ along with $\mathrm{Chl} b$ and carotenoids (Car) are found throughout higher plants, extremely different $\mathrm{Chl} a / b$ ratios have been reported in many plant and algae mutants (Thornber and Highkin 1974, Eskins et al. 1983, Rühle et al. 1983, Yang et al. 1990, Zhang et al. 2011). Such Chl-deficient mutants have been extensively used to study the Chl biosynthetic pathway and the biogenesis of the photosynthetic apparatus in barley, maize, pea, sweet clover, wheat, rice, soybean, sugar beet, Arabidopsis, and C. reinhardtii (Chen et al. 2007, Chu et al. 2015, Bujaldon et al. 2017, Zhu et al. 2019).

Rice (Oryza sativa L.) is a major crop worldwide. However, the current rice production is insufficient for meeting the world food demand, owning to continuous increases in world population. Consequently, great efforts have been made to enhance the yield of these plants (Gupta 2013). Studies have shown that photosynthesis can affect rice yield (Ambavaram et al. 2014). Enhancing the $\mathrm{Chl}$ contents was reported to have positive effects on biomass, photosynthetic rate, and grain yield in rice (Wang et al. 2008). In fact, such studies have described at least 16 different Chl-deficient rice mutants, which based on Chl $a / b$ ratios, have been separated into two main types: Type 1 , in which $\mathrm{Chl} b$ is completely absent (Chl $b$-lacking mutants), and Type 2, which possesses $\mathrm{Chl} a / b$ ratios of $\sim 10$ (Type $2 \mathrm{a}$ ) or $\sim 15$ (Type 2b) (Terao et al. 1985a,b; 1996). However, the mechanisms that underlie differences

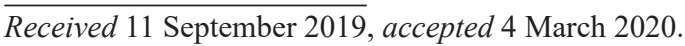

${ }^{+}$Corresponding author; e-mail: cmyang@gate.sinica.edu.tw (C.M. Yang), wendar@ntu.edu.tw (W.D Huang)

Abbreviations: Car - carotenoids; ch1 - Chlorina 1 mutant rice; Chl - chlorophyll; DEGs - different expressed genes; GO - gene ontology; $w t$ - Norin No. 8 wildtype rice.

Acknowledgments: This study was supported by the Taiwan International Graduated Program (TIGP) of National Taiwan Normal University (NTNU), in cooperation with the Biodiversity Research Center (BRC) of the Academia Sinica, Taiwan. The authors are grateful to three anonymous reviewers for their valuable evaluation, suggestions, and comments which helped us improve the manuscript. 
between the Chl $a / b$ of Chl-deficient and wildtype rice remain unclear. Currently, the use of next-generation sequencing (NGS) for transcriptome profiling is widely used for the broad assessment of gene expression. In the present study, wildtype (Norin No. 8, wt) rice (Oryza sativa L.) and a Chl $b$-lacking mutant of wt (Chlorina 1, chl), which were provided by Dr. Tomio Terao, were used to investigate the effects of gene expressional patterns on Chl regulation in chl rice. This study compared the characterization, differentially expressed genes (DEGs), and transcription factors (TFs) related to Chl biosynthesis and degradation between $w t$ and $c h 1$ rice. This work is the first to report molecular mechanism underlying $\mathrm{Chl} a / b$ ratio of a $\mathrm{Chl} b$-lacking mutants in rice.

\section{Materials and methods}

Plant materials: Seeds of wildtype (Norin No. $8, w t$ ) and its $\mathrm{Chl} b$-lacking mutant (Chlorina $1, c h l)$ rice were graciously provided by Dr. Tomio Terao (Department of Applied Physiology, National Institute of Agrobiological Resources, Tsukuba Science City, Japan). The ch1 and $w t$ rice represent Chl-deficient mutant Type 1 and its wild type, respectively, as described above. The seeds were sown in the greenhouse, and the seedlings were grown for three weeks. Leaves were then collected, immediately frozen in liquid nitrogen, and stored at $-80^{\circ} \mathrm{C}$ for further experiments.

Chl and Car measurement: The leaf material was extracted using $80 \%(\mathrm{v} / \mathrm{v})$ acetone, according to the method described by Yang et al. (1998). The absorbance of the extract was then measured at $480 \mathrm{~nm}$ using UV-Vis spectrophotometer (Hitachi U2800, Tokyo, Japan) at room temperature to determine total $\mathrm{Chl}$ content, Car content, and $\mathrm{Chl} a / b$ ratio.

Ultrastructure microscope: Wildtype and chl leaf tissues were trimmed into small cubes (approx. $0.5 \times 0.5 \times$ $0.5 \mathrm{~mm}$ ). The tissue pieces were immersed in $2.5 \%$ glutaraldehyde at $4{ }^{\circ} \mathrm{C}$ for $24 \mathrm{~h}$ followed by $1 \% \mathrm{OsO}_{4}$ for $2 \mathrm{~h}$. The tissues were trimmed to $70 \mathrm{~nm}$ using a Leica EM UC6 ultramicrotome (Leica Microsystems GmbH, Wetzlar, Germany), stained using 1\% (w/v) uranyl acetate and $1 \%(\mathrm{w} / \mathrm{v})$ lead citrate (Spurr 1969), and then visualized using a Phillip Tecnai 12 transmission electron microscope (JEOL Ltd., Japan).

Transcriptome sequencing: For transcription analysis, total RNA was extracted from $w t$ and chl leaves using the RNeasy Plant Mini Kit (Qiagen, Hilden, Germany), following the manufacturer's instructions. For transcriptome sequencing, paired-end cDNA libraries for the $w t$ and chl rice were constructed, and the resulting cDNA libraries were sequenced using BGISEQ-500 platform (Beijing Genomics Institute, Shenzhen, China). Adapter sequences, ambiguous nucleotides, and low quality sequences were trimmed from the raw reads using SOAP nuke v. 1.5.2 (https://github.com/BGI-flexlab/SOAPnuke). Both the cDNA library construction and transcriptome sequencing were performed by a commercial service provider (Tri-I Biotech, Inc., New Taipei City, Taiwan).

Transcriptome analysis: The clean reads were mapped to the genome of $O$. sativa 'Nipponbare' (http://rapdb.dna. affrc.go.jp/) using hierarchical indexing for the spliced alignment of transcripts in HISAT2 v. 2.0.4 (http://www. ccb.jhu.edu/software/hisat). Gene expression analysis was performed using Bowtie2 v. 2.2 .5 (http://bowtie-bio. sourceforge.net/Bowtie/index.shtml), and gene expression levels were calculated using RSEM v. 1.2.12 (http:// deweylab.biostat.wisc.edu/RSEM). Pearson's correlation, hierarchical clustering and illustration of the samples were performed using the cor, hclust, and ggplot 2 functions of $R$, respectively. Meanwhile, DEGs were detected using PossionDis, which is based on Poisson distribution with parameters of Fold Change $\geq 2.00$ and FDR $\leq 0.001$ (Audic and Claverie 1997), and were subject to Gene Ontology (GO) and $K E G G$ pathway analysis.

Quantitative RT-PCR (qPCR): Total RNA $(\mu \mathrm{g})$ extracted from $w t$ and chl rice was subjected to cDNA synthesis using a Transcriptor First Strand cDNA Syn-thesis Kit (Roche Diagnostic Systems, Branchburg, NJ, USA) and oligo (dT) primer. Primer sets were designed using Primer Premiere 6 software (Premiere Biosoft, Palo Alto, USA) (Table 1S, supplement). qPCR was performed using the StepOne Plus Real-Time PCR system (Applied Biosystems, Life Technologies Inc., Italy) with Roche FastStar Universal SYBR Green Master reagent (Roche Diagnostic Systems, Branchburg, NJ, USA). Relative gene expression values were calculated as $2^{-\Delta \mathrm{Ct}}$, where $\Delta \mathrm{Ct}$ was calculated as the difference between the target gene $\mathrm{Ct}$ and reference gene Ct. The fold change of each gene in leaf tissues was calculated by $2^{-\Delta \mathrm{Ct}}{ }_{\text {mutant }} / 2^{-\Delta \mathrm{Ct}}$ wildtype.

Statistical analysis: The pigment contents and relative gene expression (qPCR results) of $w t$ and $c h 1$ rice were statistically analyzed using the least significant difference (LSD) $t$-test at $p \leq 0.05$, which was performed by $S A S v .8 .0$ (Research Triangle Park, NC, USA).

\section{Results}

Characterization of $\boldsymbol{c h} \mathbf{1}$ rice: The $w t$ leaves were dark green, whereas $c h 1$ leaves ranged from light green to green (Fig. 1A). Overall, the $w t$ leaves accumulated about twice as much of Chl (total Chl) as in the chl leaves $(\sim 91 \%)$ and $\sim 78 \%$ more Car. Furthermore, Chl $b$ was absent in the $c h 1$ leaves, thereby yielding a $\mathrm{Chl} a / b$ ratio of $\infty$ so only $\mathrm{Chl} a$ was present (Fig. 1B). Chloroplasts from the mesophyll cells of $w t$ leaves exhibited normal structure, including distinct thylakoid membranes and stromal lamellae with small starch granules and one or two plastoglobuli (Fig. $2 A, B$ ). By contrast, those of $c h 1$ leaves were presented as indistinct thylakoid membranes, indistinct or absent stromal lamellae, and abundant vesicles and plastoglobuli (Fig. 2C). The stacked thylakoid grana of the chl leaves were also thinner than those of $w t$ leaves (Fig. 2D). 

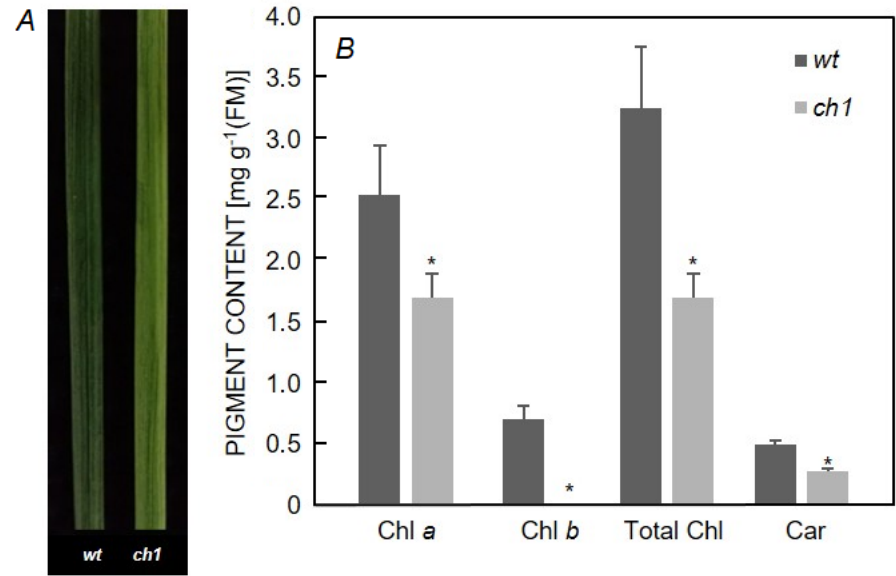

Fig. 1. Leaf coloration and determination of pigments content. (A) Leaf coloration of wildtype (wt) and Chl $b$-lacking $(c h 1)$ rice. $(B)$ Chlorophyll (Chl) and carotenoid (Car) contents of wildtype (wt) and Chl $b$-lacking (chl) rice. Asterisks indicate significant differences between the pigment contents of the $w t$ and $c h 1$ rices $(p \leq 0.05)$.
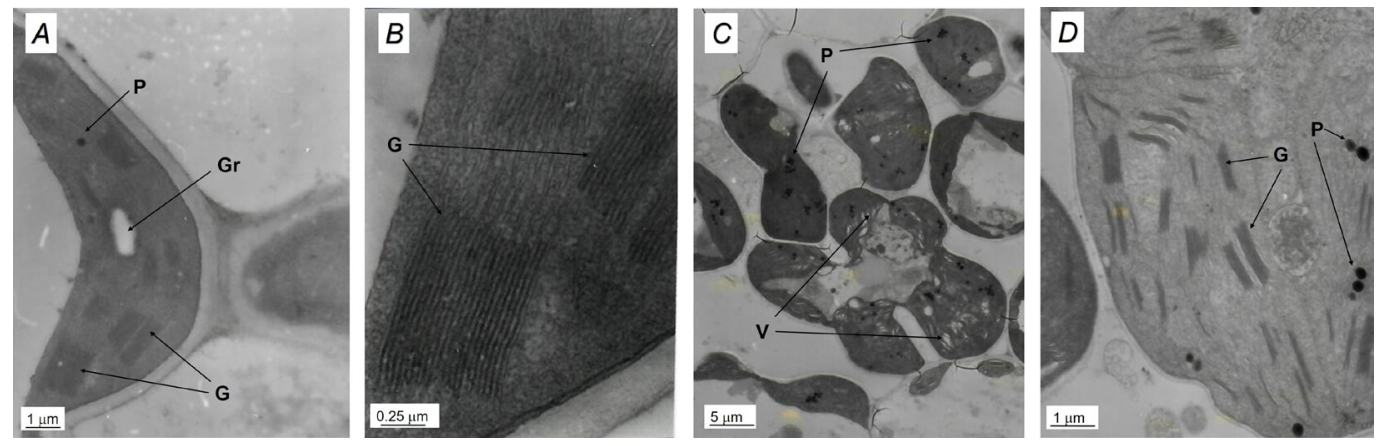

Fig. 2. Chloroplast ultrastructure of wildtype and chlorophyll $b$-lacking rice. $(A, B)$ Normal chloroplast structure of wildtype (wt) rice. $(C, D)$ Abnormal chloroplast structure chlorophyll $b$-lacking $(c h l)$ rice, showing indistinct thylakoid membranes and abundant plastoglobuli. G - grana; P - plastoglobuli; Gr - granulose; V - vacuole.

Genome mapping and gene expression: Approximately 1.27-1.45 GB of clean reads were produced using the BGISEQ-500 platform, and after filtering, 25.31-28.93 million reads were mapped to the $O$. sativa 'Nipponbare' reference genome (http://rapdb.dna.affrc.go.jp/), with match ratios in the range of $96.84-97.94 \%$ (Table $2 \mathrm{~S}$, supplement). A total of 93.0 and $89.67 \%$ of the $w t$ and ch1 libraries, respectively, were mapped to the reference genome, whereas the 80.91 and $80.42 \%$ of the libraries were mapped to single location in the reference genome (Table 3S, supplement). Approximately $89.6 \%$ of genes expressed by $w t$ and chl rice were detected in both (Fig. 1S).

\footnotetext{
Annotation and analysis of different gene expression DEGs: Functional annotation was performed to obtain information protein function annotation, pathway annotation and gene ontology (GO) annotation. Genes with sequence orientation were aligned against $K E G G$ database. GO is an international standardized gene functional classification system in which transcripts are attributed to biological processes, cellular components, or molecular function. Fig. 3 shows the distribution of genes among the GO categories. In total, 22,493 genes were mapped, with 7,972 genes assigned to 'biological process', 10,282 genes assigned to 'cellular component', and 4,239 genes
}

assigned to 'molecular function'. Individual genes can be assigned to multiple GO-terms (Fig. 3).

DEGs were identified using the fragments per kb per million reads (FPKM) method, and the Poisson distribution equation, with false discovery rate (FDR) $\leq 0.001$ and absolute $\log 2$ ratio $\leq 1$ (Fig. 4). Total of 1,971; 8,336 ; and 15,886 genes were identified as upregulated, downregulated, and unaffected in chl leaves, respectively (Fig. 4A).

Role of Chl metabolism genes in leaf coloration: DEGs related to chloroplast development and division were identified based on KEGG pathway annotation. Genes related to the FtsZ cell division protein genes and PsbR chloroplast photosystem II protein genes were downregulated in the chl rice. Meanwhile, PetF genes related to the FDX electron transfer protein genes were greatly upregulated (Table 1).

A total of 19 DEGs related to Chl biosynthesis and four DEGs related to $\mathrm{Chl}$ degradation were identified based on KEGG pathway annotation. The expression levels of the DEGs were determined using hierarchical cluster analysis (Fig. 5). A UROD homolog (Osa_4326635), CPOX (Osa_4336951), and $\mathrm{MgCH}$ (Osa_4334537) were significantly downregulated in $\operatorname{ch} 1(\Delta \log \overline{2}=2.1,2.2$, and 2.4, respectively) as compared to the $w t$ (Fig. 5). These 


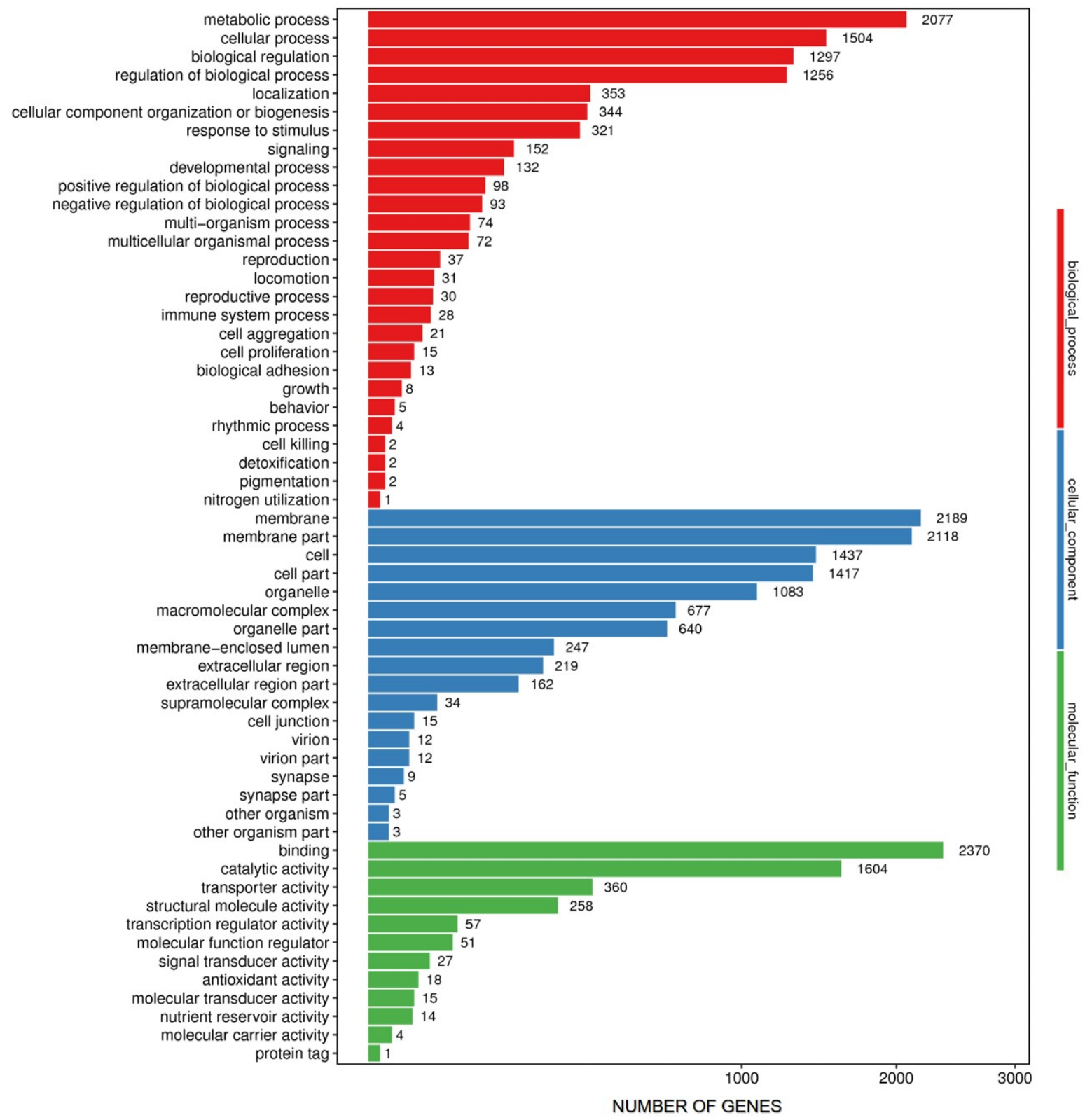

Fig. 3. Gene Ontology classification of differentially expressed genes from wildtype (wt) and chlorophyll $b$-lacking $(c h l)$ rice.

results suggested that the Chl biosynthesis of chl was similar to that of $w t$ rice, even though the $\operatorname{chl}$ rice exhibited the significantly lower Chl content (Fig. 1).

Role of transcription factors (TFs) in leaf coloration: TFs are key regulatory proteins that play important roles in the regulation of gene expression. In the present study, 671 DEGs were identified as putative TFs and were associated with a total of $51 \mathrm{TF}$ families. The most abundant $\mathrm{TF}$ family was the MYB superfamily (90 DEGs), followed by the MYB-related (62 DEGs), bHLH (48 DEGs), WRKY (46 DEGs), AP2-EREBP (40 DEGs), and NAC (35 DEGs) TF families (Table 2). The MYB, MYB-related, WRKY, and NAC families were represented by $35,28,21$, and
28 DEGs, respectively, which were downregulated in ch1 rice. Meanwhile, 19 DEGs were associated with the HLH TFs, most of which were also significantly downregulated in the ch1 rice, and some of the DEGs which were associated with the AP2-EREBP, C2H2, and TCP families were downregulated in the $c h 1$ rice, as well (Table 2).

DEGs validation: To validate the identification of DEGs related to $\mathrm{Chl}$ biosynthesis and degradation, different expression analysis of the identified DEGs was performed. Eleven of the twelve genes analyzed by qPCR expression patterns were similar to those observed using transcription analysis (Fig. 6). More specifically, UROD, CPOX, and $\mathrm{MgCH}$ were significantly upregulated in $c h 1$, whereas 

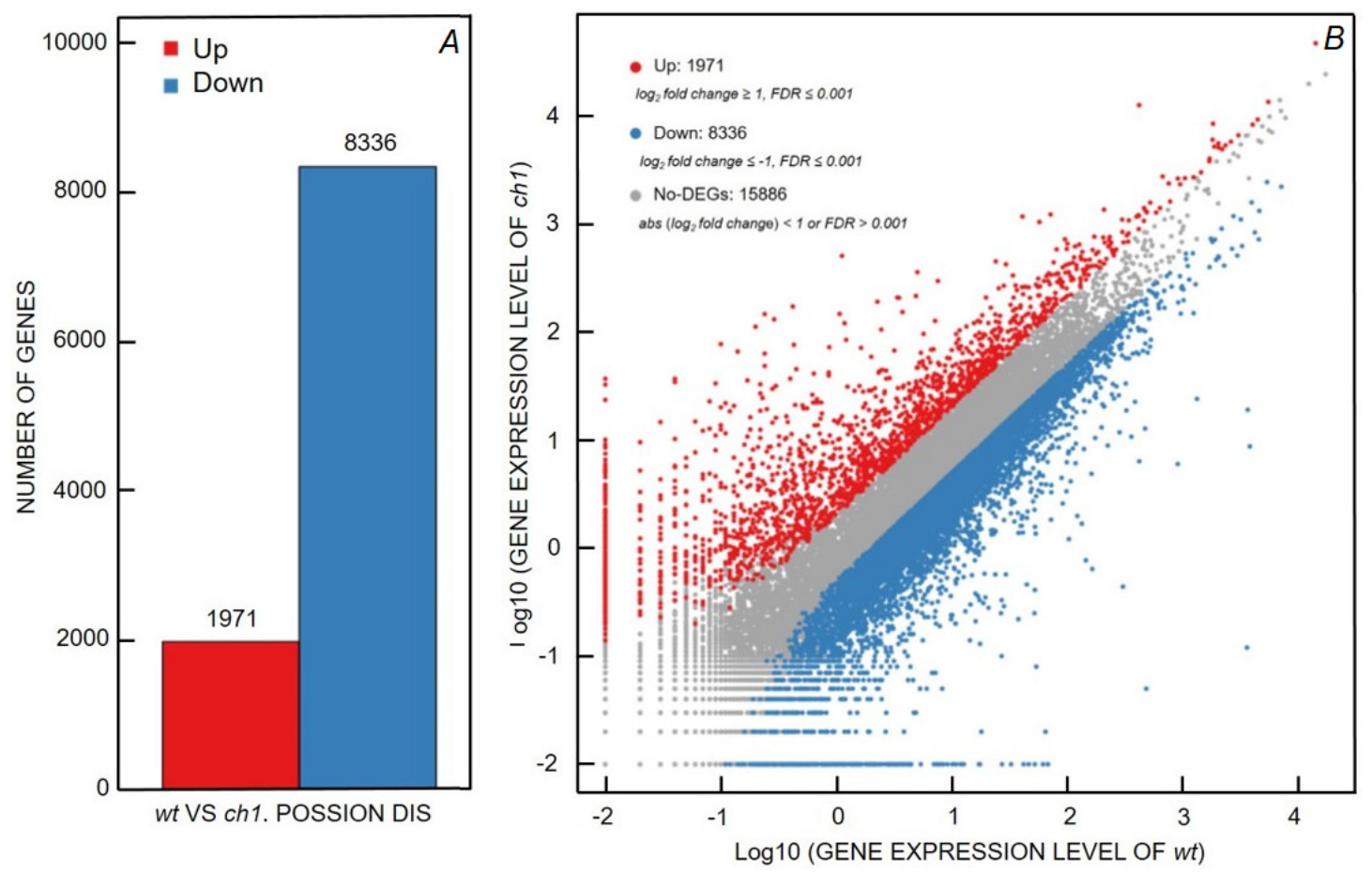

Fig. 4. Differentially expressed genes (DEGs) from wildtype (wt) and chlorophyll b-lacking $(c h 1)$ rice. (A) Comparison of upregulated and downregulated DEGs. $(B)$ Classification of genes, based on differences in expression level.

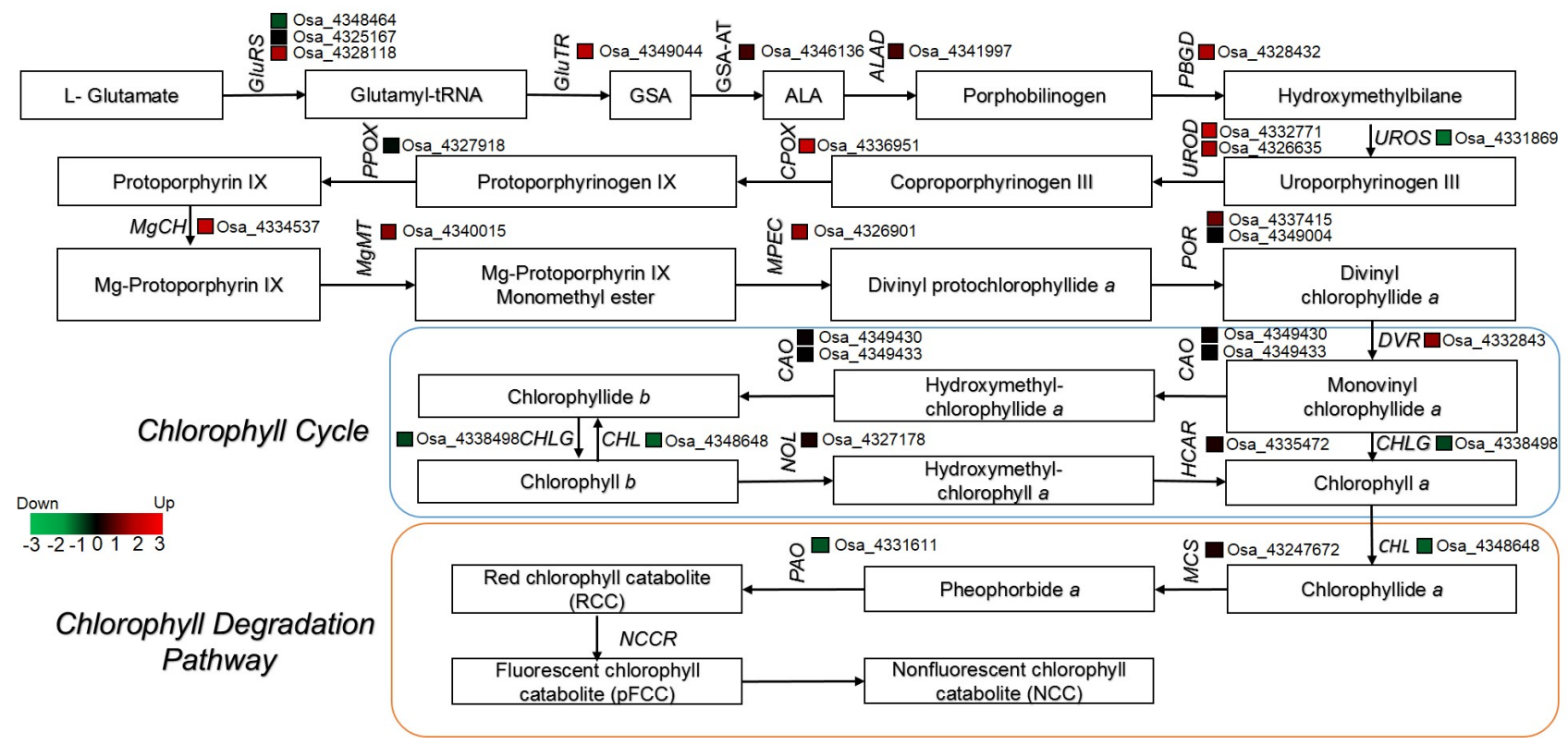

Fig. 5. Expression profiles of differentially expressed genes involved in chlorophyll biosynthesis and degradation. The expression levels of the chlorophyll $b$-lacking $(c h l)$ are compared to those of wildtype $(w t)$ rice.

GluRS was significantly downregulated. However, in contrast to the results of transcription analysis, the qPCR results indicated that NOL was significantly upregulated in ch1 (Fig. 6). In general, the qPCR results were consistent with the RNA-Seq data, despite some differences in expression level.

\section{Discussion}

Physiological changes and pigment contents analysis: In higher plants, Chl biosynthesis and degradation are involved in leaf coloration. Therefore, Chl-deficientmutants exhibit changes in leaf coloration (Yang et al. 2015b). 
Table 1. Distribution of unigenes amongst KEGG pathways that contain differentially expressed genes. TF - transcription factor; ch1 - chlorophyll b-lacking mutant (Chlorina 1).

\begin{tabular}{llllll}
\hline Function & Gene ID & TF family & $\log _{2}$ fold change & Expression in ch1 & Annotation \\
\hline Chlorophyll & Osa_4332771 & UROD & 2.1 & Upregulated & uroporphyrinogen decarboxylase \\
biosynthesis & Osa_4336951 & CPOX & 2.2 & Upregulated & coproporphyrinogen III oxidase \\
& Osa_4334537 & $M g C H$ & 2.4 & Upregulated & Mg-chelatase \\
Photosynthesis & Osa_107276047 & PsbR & -5.15 & Downregulated & photosystem II $10 \mathrm{kDa}$ protein \\
& Osa_4342395 & PsbR & -7.2 & Downregulated & photosystem II 10 kDa protein \\
& Osa_4335779 & PetF & 2.8 & Upregulated & ferredoxin-1 \\
& Osa_9270637 & PetF & -5.5 & Downregulated & ferredoxin, root R-B1 \\
& Osa_4333746 & PetF & 5.3 & Upregulated & ferredoxin-2 \\
Chloroplast & Osa_4338932 & FtsZ1 & -2.7 & Downregulated & Cell division protein FtsZ homolog 1 \\
division & Osa_4338932 & FtsZ2 & -2.3 & Downregulated & Cell division protein FtsZ homolog 2 \\
\hline
\end{tabular}

Chl-deficient mutants have been reported in many species, including barley, maize, peas, sweet clover, wheat, rice, rockcress, and maidenhair tree (Yang et al. 1990, Chen et al. 2007, Chu et al. 2015, Bujaldon et al. 2017, Li et al. 2018, Zhu et al. 2019). Our results are consistent with those of another study, which reported that $\mathrm{Chl} b$ was completely absent in a Chl-deficient mutant (Terao et al. 1985a). The total $\mathrm{Chl}$ and $\mathrm{Chl} b$ content of the $\operatorname{chl}$ leaves remarkably decreased as compared to $w t$ leaves. This result suggested that the reductions in total $\mathrm{Chl}$ content contributes to leaf color variation at the physiological level.

The analysis of the chloroplast ultrastructure of the $w t$ and ch1 rice revealed differences in thylakoid membrane structure. The $w t$ chloroplasts possessed typical thylakoid membranes that were efficient for harvesting and converting light energy, whereas the chl chloroplast possessed abnormal thylakoid membranes. Similarly, chloroplasts of green bamboo leaves contained abundant thylakoid membranes, whereas the thylakoid membranes of $\mathrm{Chl} b$-lacking leaves were converted into numerous abnormal vesicles (Yang et al. 2015a). In Anthurium andraeanum, the mesophyll cells of wildtype plants contained normal chloroplast which contained small starch granules and, thus, large gaps among stroma thylakoids (Yang et al. 2015b).

The results obtained in this study also demonstrated differences in the chloroplast structure of $w t$ and chl rice. The ultrastructure of chl rice (i.e., abundant plastoglobuli, indistinct thylakoid membranes, and reduced starch granules) indicated abnormal development and possibly a reduced accumulation of pigments, which would reduce light energy conversion and account for the coloration difference in the $w t$ and $c h 1$ rice. The chl rice also exhibited thinner stacked grana, caused by a reduction in the thylakoid membranes per granum. Therefore, the Chl $a / b$ ratio of $c h 1$ rice may reflect the abnormal plastid development and function of plastid in the chl rice.

Chloroplast-related DEGs and photosynthetic capacity: The normal development of chloroplasts in higher plants requires the coordination of both plastid nuclear genes, and changes in the expression levels of either gene type could affect the biogenesis in Chl metabolism and chloroplast assembling, thus affecting $\mathrm{Chl} a / b$ ratio and leaf coloration (Li et al.2015, Yang et al.2015a). FtsZ proteins, which play important roles in cell and organelle division, also regulate chloroplast division (Schmitz et al. 2009). The expression levels of Fts Z1 and FtsZ2 were significantly downregulated in the chl rice, which indicated that chloroplast division may be defective. This finding was strongly supported by differences in the chloroplast ultrastructure and leaf coloration of $w t$ and $c h 1$ rice, thereby suggesting that the lower accumulation of $\mathrm{Chl}$ contents was responsible for the lower $\mathrm{Chl} b$ content and higher $\mathrm{Chl} a / b$ ratio of the $c h 1$ rice. During photosynthesis, light energy is captured by pigments in the LHC proteins, which then transfer absorbed light energy to PSI and PSII reaction center complexes (Goral et al. 2012, Zhao et al. 2017). In a previous study of a Chl-deficient $A$. thaliana mutant, LHC protein was strongly reduced, or even completely absent, thereby impairing the grana stacking of the plant's chloroplast (Kim et al. 2009). The multi-protein and pigment complex known as PSII provides the high redox potential needed to oxidize water, and contains more than 20 subunits, including PsbR (Shi et al. 2012). Chloroplast FDX proteins including PetF, play important roles in electron transfer. Enhancing PetF genes raises reduced ascorbate contents and lowers $\mathrm{H}_{2} \mathrm{O}_{2}$ contents under natural conditions in C. reinhardtii (Lin et al. 2013). Moreover, reducing FDX causes leaf yellowing in transgenic plants grown under high-light conditions (Holtgrefe et al. 2003, Hanke and Hase 2008). In the present study, two DEGs (PsbR) were identified as the members of the LHCII gene family, and their expression levels were strongly downregulated in the ch1 rice, which might indicate that the amounts of lightharvesting $\mathrm{Chl}$ proteins were reduced, thereby lowering the photosynthetic capacity. Furthermore, PetF genes were strongly upregulated in the $c h 1$ rice, which might enhance Chl degradation (Table 1).

RNA-Seq analysis and Chl-related DEGs: Both NGS and transcriptome profiling provide information about DEGs (Seo et al. 2004, Wang et al. 2009). Therefore, the combination of RNA-Seq technology and improved 
M.K. NGUYEN et al.

Table 2. Summary of differentially expressed gene transcription factor genes of chlorophyll b-lacking (chl) rice compared to wildtype $(w t)$.

\begin{tabular}{|c|c|c|c|}
\hline Transcription factor family & Total no. genes & No. genes upregulated & No. genes downregulated \\
\hline zf-HD & 5 & 0 & 1 \\
\hline WRKY & 46 & 2 & 21 \\
\hline VOZ & 1 & 0 & 0 \\
\hline TUB & 5 & 0 & 3 \\
\hline Trihelix & 17 & 0 & 1 \\
\hline TIG & 2 & 0 & 0 \\
\hline Tify & 8 & 0 & 1 \\
\hline TCP & 6 & 1 & 2 \\
\hline SRS & 3 & 0 & 2 \\
\hline Sigma70-like & 3 & 0 & 0 \\
\hline SBP & 5 & 0 & 3 \\
\hline S1Fa-like & 1 & 0 & 0 \\
\hline PLATZ & 2 & 0 & 1 \\
\hline PBF-2-like & 2 & 0 & 0 \\
\hline OFP & 8 & 3 & 0 \\
\hline NAC & 35 & 3 & 28 \\
\hline MYB-related & 62 & 12 & 25 \\
\hline MYB & 90 & 17 & 35 \\
\hline mTERF & 23 & 1 & 14 \\
\hline MADs & 16 & 0 & 11 \\
\hline LOB & 1 & 0 & 0 \\
\hline LIM & 4 & 0 & 0 \\
\hline HSF & 9 & 0 & 5 \\
\hline HB & 2 & 1 & 0 \\
\hline GRF & 4 & 0 & 0 \\
\hline GRAS & 18 & 0 & 8 \\
\hline $\mathrm{GeBP}$ & 3 & 0 & 0 \\
\hline G2-like & 23 & 6 & 15 \\
\hline FHA & 9 & 0 & 3 \\
\hline FAR1 & 18 & 0 & 4 \\
\hline E2F-DP & 3 & 0 & 0 \\
\hline DBP & 1 & 0 & 0 \\
\hline CSD & 1 & 0 & 0 \\
\hline СРP & 6 & 0 & 3 \\
\hline CAMTA & 3 & 0 & 0 \\
\hline $\mathrm{C} 3 \mathrm{H}$ & 19 & 3 & 2 \\
\hline $\mathrm{C} 2 \mathrm{H} 2$ & 20 & 1 & 7 \\
\hline C2C2-YABBY & 5 & 1 & 0 \\
\hline C2C2-GATA & 11 & 2 & 2 \\
\hline C2C2-Dof & 11 & 0 & 6 \\
\hline $\mathrm{C} 2 \mathrm{C} 2-\mathrm{CO}-$ like & 10 & 2 & 1 \\
\hline b2IP & 16 & 2 & 1 \\
\hline BSD & 7 & 0 & 1 \\
\hline bHLH & 48 & 4 & 18 \\
\hline BES1 & 2 & 0 & 1 \\
\hline $\mathrm{BBR} / \mathrm{BPC}$ & 2 & 0 & 2 \\
\hline ARR-B & 4 & 0 & 4 \\
\hline $\mathrm{ARF}$ & 14 & 0 & 4 \\
\hline AP2-EREBP & 40 & 2 & 8 \\
\hline Alfin-like & 12 & 0 & 1 \\
\hline ABI3VP1 & 15 & 0 & 5 \\
\hline
\end{tabular}




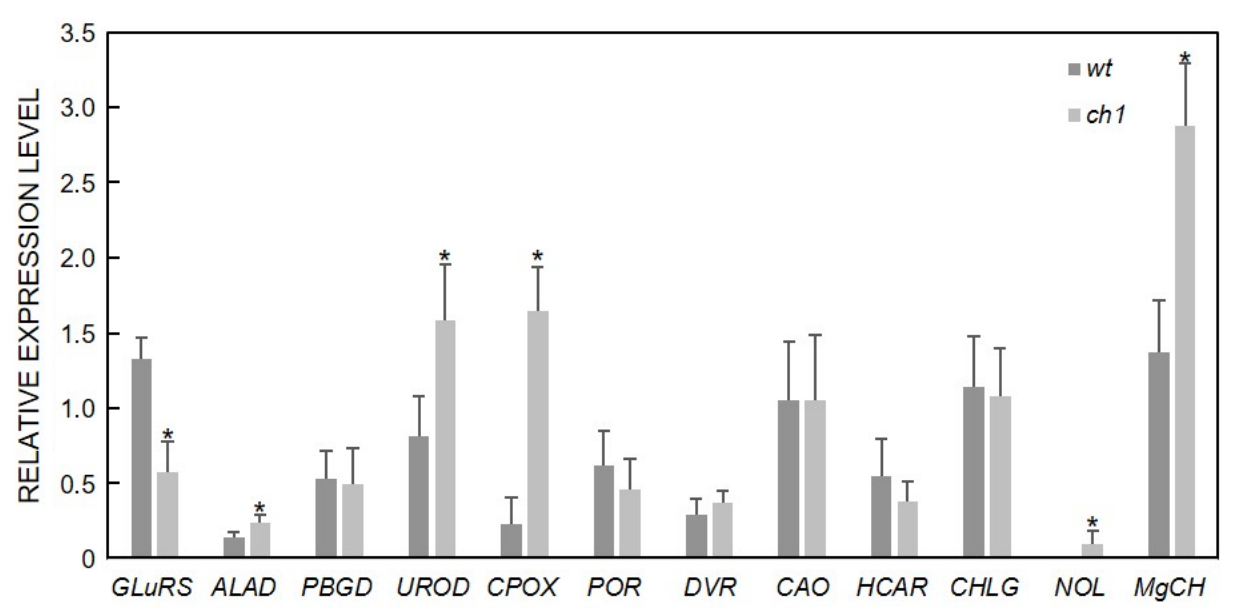

Fig. 6. Expression of levels of 12 differentially expressed genes from wildtype $(w t)$ and chlorophyll $b$-lacking (chl) rice. Gene expression was measured using quantitative real-time PCR. Asterisks indicate significant differences between the expression levels of the $w t$ and $c h 1$ rices $(p \leq 0.05)$. analysis methods enables the recognition of novel transcript isoforms and the estimation of their roles in altering $\mathrm{Chl} a / b$ ratios. In the present study, transcriptome profile analysis of $w t$ and $c h 1$ rice revealed a total of 26,193 DEGs. It is worth noting that some of DEGs related to Chl biosynthesis and degradation, chloroplast development, photosynthesis, and pigment biosynthesis are likely contributing to the observed differences in $\mathrm{Chl} a / b$ ratios.

$\mathrm{Chl}$ is responsible for light harvesting and transferring in antenna systems, and for charge separation and electron transport in reaction center (Ben-Shem et al. 2003, Nelson and Yocum 2006). In A. thaliana, 27 genes were identified to encode 15 Chl-biosynthesis enzymes (Meier et al. 2011), and changes in the expression could generate Chlmetabolism disorders that alter $\mathrm{Chl} a / b$ ratio. In common pathway of $\mathrm{Chl}$ biosynthesis metabolism, changes in the expression of GluRS could affect Chl contents (Fang et al. 2016). Uroporphyrinogen decarboxylase (UROD), which is a branch point enzyme in the biosynthesis of tetrapyrroles, catalyzes the decarboxylation of four acetate groups of uroporphyrinogen III, thereby yielding coproporphyrinogen III, which then contributes to heme and Chl biosynthesis (Fan et al. 2007). Moreover, Mg-chelatase deficiency is a common factor among many Chl-deficient mutants. For example, the downregulation of $\mathrm{MgCH}$ genes reduce $\mathrm{Mg}$-chelatase activity in $C$. sinensis (Wang et al. 2014).

In the present study, a total of 19 DEGs related to Chl biosynthesis were identified based on KEGG pathway annotation. Among these DEGs, GluTR was downregulated in the chl rice. However, UROD and CPOX were upregulated, an observation that was further confirmed through qPCR, and that suggested that later stages of Chl biosynthesis occurred in the chl rice, whereas early stages were inhibited. Moreover, the expression of $\mathrm{MgCH}$ was upregulated due to less early stage products which suggests that the Chl contents of the $c h 1$ rice needs to be enhanced in order to adapt to light-harvesting needs for conversion to sugar. In addition, the expression of Chl cycle- and degradation-related genes, including $C A O$, $C H L G, H C A R, C H L, M C S$, and $P A O$, were insignificantly different. Non-yellow coloring1 (NYC1) is a stay-green rice mutant in which degradation of Chl during senescence is impaired. Pigment analysis has revealed that degradation of both Chls and carotenoids is repressed in the NYC1, in which most LHCII isoforms are selectively retained during senescence. The NOL protein is closely related to $N Y C 1$, and the upregulation of $N O L$ has been reported to drastically reduce the Chl $b$ content (Kusaba et al. 2007, Sato et al. 2009). In the present study, qPCR analysis indicated that $N O L$ was significantly upregulated in $c h 1$ rice, although the transcriptome data indicated that it was unaffected. The Chl content of $c h 1$ rice was significantly reduced as compared to $w t$ rice. This strongly suggests that the abnormal chloroplast development of the $\operatorname{ch} 1$ rice affects the accumulation of $\mathrm{Chl} b$ and that, together with the significant upregulation of $N O L$ genes, enhances the degradation of Chl $b$ to $\mathrm{Chl} a$. This is similar to observation in G. biloba leaves, where the upregulation of $N O L / N Y C$ genes was associated with coloration changes from green to light-green (Li et al. 2018).

There are also two other possible explanations for the absence of $\mathrm{Chl} b$ in $c h 1$ rice. First, because chlorophyllide $a$ oxygenase (CAO) is the main enzyme involving to the conversion of Chl $a$ to $\mathrm{Chl} b$ in Chl cycle (Reinbothe et al. 2006, Yang et al. 2015c). The expression of CAO genes plays an important role in the $\mathrm{Chl} a / b$ ratio. In this study, both the transcriptome and qPCR analysis confirmed that there was no significant difference between the $C A O$ expression of the $w t$ and chl rice, which suggests that $C A O$ expression was blocked at the translation level, or that abnormal translation contributed to the formation of nonfunctional CAO proteins. Secondly, many studies reported that the conversion of $\mathrm{Chl} b$ to Chl $a$ via hydroxymethyl $\mathrm{Chl} a$ is the first step of $\mathrm{Chl} b$ degradation, and is catalyzed by NOL (Ito et al. 1996, Horie et al. 2009). In addition, NOL has multi-substrate specificity and is unbound to light-harvesting complexes including $\mathrm{Chl} b$, chlorophyllide $b$, pheophorbide $b$, and pheophytin $b$ (Shimoda et al. 2012). However, pheophytin $b$ and pheophorbide $b$ were reported not to be produced in chloroplast due to the specificity of $\mathrm{Mg}$-dechelatase (Tanaka and Tanaka 2019). In contrast, another study reported that pheophorbide $b$ is accumulated during cell death after incubating a plant whose core antenna complex contained Chl $b$ in dark conditions (Shimoda et al. 2012). 
Therefore, the mechanism underlying pheophorbide $b$ presence in plant cells is still unclear. We hypothesized that $\mathrm{Chl} b$ might be degraded via the pheophorbide $b$ pathway in ch1 rice, however, this requires further investigations. The absence of Chl $b$ in $c h 1$ may confirm that the pheophorbide $b$ is capable of degrading $\mathrm{Chl} b$, which is a pathway that remains poorly understood.

This study also found that grana stacking is normal along with the absence of Chl $b$ and reduced levels of Chl $a$. Similar patterns have been reported by previous studies, which suggests that stacking grana affects both LHCII protein and another factor (Nakatani and Baliga 1985, Ouijja et al. 1988, Yang and Chen 1996). Accordingly, the results of the present study provide strong evidence to support the conclusion that other than Chl-deficiency-mediated changes in $\mathrm{LCH}$ proteins are involved in grana stacking.

Conclusion: This study reported the mechanisms underlying the different $\mathrm{Chl} a / b$ ratios in wildtype rice and Chl $b$-lacking mutant (chl) rice. Lower Chl content and abnormal chloroplasts structure were observed in the ch1 leaves. Transcription analysis identified 23 DEGs and 671 TFs, which are involved in Chl metabolism, chloroplast development, cell division, and photosynthesis. qPCR analysis was used to validate the DEGs. Changes in the expression of genes related to chloroplast development, $\mathrm{Chl}$ biosynthesis, and degradation reduced the accumulation of $\mathrm{Chl}$ in the $c h 1$ rice. This result suggest that the $\mathrm{Chl} a / b$ ratio was amplified both by reduction in $\mathrm{Chl}$ accumulation, owing to abnormal chloroplast development, and by the enhanced conversion of $\mathrm{Chl} b$ to $\mathrm{Chl} a$. Moreover, the results of the present study indicated the same Chl-cycle pattern in the wildtype and chl rice, which suggests the involvement of either abnormal $C A O$ translation, or another $\mathrm{Chl} b$ degradation pathway. This study provides insights into the molecular mechanism that underlies different $\mathrm{Chl} a / b$ ratios in rice.

\section{References}

Ambavaram M.M.R., Basu S., Krishnan A. et al.: Coordinated regulation of photosynthesis in rice increases yield and tolerance to environmental stress. - Nat. Commun. 5: 5302, 2014.

Audic S., Claverie J.M.: The significance of digital gene expression profiles. - Genome Res. 7: 986-995, 1997.

Ben-Shem A., Frolow F., Nelson N.: Crystal structure of plant photosystem I. - Nature 426: 630-635, 2003.

Bujaldon S., Kodama N., Rappaport F. et al.: Functional accumulation of antenna proteins in chlorophyll $b$-less mutants of Chlamydomonas reinhardtii. - Mol. Plant 10: 115-130, 2017.

Chen X., Zhang W., Xie Y. et al.: Comparative proteomics of thylakoid membrane from a chlorophyll $b$-less rice mutant and its wild type. - Plant Sci. 173: 397-407, 2007.

Chu P., Yan G.X., Yang Q. et al.: iTRAQ-based quantitative proteomics analysis of Brassica napus leaves reveals pathways associated with chlorophyll deficiency. - J. Proteomics 113: 244-259, 2015.

Eckhardt U., Grimm B., Hörtensteiner S.: Recent advances in chlorophyll biosynthesis and breakdown in higher plants. Plant Mol. Biol. 56: 1-14, 2004.
Eskins K., Delmastro D., Harris L.: A comparison of pigmentprotein complexes among normal, chlorophyll-deficient and senescent soybean genotypes. - Plant Physiol. 73: 51-55, 1983.

Fan J., Liu Q., Hao Q. et al.: Crystal structure of uroporphyrinogen decarboxylase from Bacillus subtilis. - J. Bacteriol. 189: 3573-3580, 2007.

Fang Y., Zhao S., Zhang F. et al.: The Arabidopsis glutamyltRNA reductase (GluTR) forms a ternary complex with FLU and GluTR-binding protein. - Sci. Rep.-UK 6: 19756, 2016.

Fromme P., Melkozernov A., Jordan P., Krauss N.: Structure and function of photosystem I: interaction with its soluble electron carriers and external antenna systems. - FEBS Lett. 555: 40-44, 2003.

Goral T.K., Johnson M.P., Duffy C.D.P. et al.: Light-harvesting antenna composition controls the macrostructure and dynamics of thylakoid membranes in Arabidopsis. - Plant J. 69: 289-301, 2012.

Gupta J.: Climate change and water law: The regimes compared. In: Grover V.I. (ed.): Impact of Climate Change on Water and Health. Pp. 30-45. CRC Press, Boca Raton-London-New York 2013.

Hanke G.T., Hase T.: Variable photosynthetic roles of two leaf-type ferredoxins in Arabidopsis, as revealed by RNA interference. - Photochem. Photobiol. 84: 1302-1309, 2008.

Holtgrefe S., Bader K.P., Horton P. et al.: Decreased content of leaf ferredoxin changes electron distribution and limits photosynthesis in transgenic potato plants. - Plant Physiol. 133: 1768-1778, 2003.

Horie Y., Ito H., Kusaba M. et al.: Participation of chlorophyll $b$ reductase in the initial step of the degradation of lightharvesting chlorophyll $a / b$-protein complexes in Arabidopsis.J. Biol. Chem. 284: 17449-17456, 2009.

Huang J., Qin F., Zang G. et al.: Mutation of OsDET1 increases chlorophyll content in rice. - Plant Sci. 210: 241-249, 2013.

Ito H., Ohtsuka T., Tanaka A.: Conversion of chlorophyll $b$ to chlorophyll $a$ via 7-hydroxymethyl chlorophyll. - J. Biol. Chem. 271: 1475-1479, 1996.

Jin E., Yokthongwattana K., Polle J.E.W., Melis A.: Role of the reversible xanthophyll cycle in the photosystem II damage and repair cycle in Dunaliella salina. - Plant Physiol. 132: 352-364, 2003.

Kim E.H., Li X.P., Razeghifard R. et al.: The multiple roles of light-harvesting chlorophyll $a / b$-protein complexes define structure and optimize function of Arabidopsis chloroplasts: A study using two chlorophyll $b$-less mutants. - BBABioenergetics 1787: 973-984, 2009.

Kräutler B.: Chlorophyll breakdown and chlorophyll catabolites in leaves and fruit. - Photoch. Photobio. Sci. 7: 1114-1120, 2008.

Kusaba M., Ito H., Morita R. et al.: Rice Non-Yellow Coloring1 is involved in light-harvesting complex II and grana degradation during leaf senescence. - Plant Cell 19: 1362-1375, 2007.

Li W., Yang S., Lu Z. et al.: Cytological, physiological, and transcriptomic analyses of golden leaf coloration in Ginkgo biloba L. - Hortic. Res. 5: 12, 2018.

Li Y., Zhang Z., Wang P. et al.: Comprehensive transcriptome analysis discovers novel candidate genes related to leaf color in a Lagerstroemia indica yellow leaf mutant. - Genes Genom. 37: 851-863, 2015.

Lin Y.H., Pan K.Y., Hung C.H. et al.: Overexpression of ferredoxin, PETF, enhances tolerance to heat stress in Chlamydomonas reinhardtii. - Int. J. Mol. Sci. 14: 2091320929, 2013.

Liu W., Fu Y., Hu G. et al.: Identification and fine mapping of a thermo-sensitive chlorophyll deficient mutant in rice (Oryza 
sativa L.). - Planta 226: 785-795, 2007.

Masuda T., Fujita Y.: Regulation and evolution of chlorophyll metabolism. - Photoch. Photobio. Sci. 7: 1131-1149, 2008.

Meier S., Tzfadia O., Vallabhaneni R. et al:: A transcriptional analysis of carotenoid, chlorophyll and plastidial isoprenoid biosynthesis genes during development and osmotic stress responses in Arabidopsis thaliana. - BMC Syst. Biol. 5: 77, 2011.

Mitchell P.L., Sheehy J.E.: Supercharging rice photosynthesis to increase yield. - New Phytol. 171: 688-693, 2006.

Nakatani H.Y., Baliga V.: A clover mutant lacking the chlorophyll $a$ - and $b$-containing protein antenna complexes. Biochem. Bioph. Res. Co. 131: 182-189, 1985.

Nelson N., Yocum C.F.: Structure and function of photosystems I and II. - Annu. Rev. Plant Biol. 57: 521-565, 2006.

Ouijja A., Farineau N., Cantrel C., Guillot-Salomon T.: Biochemical analysis and photosynthetic activity of chloroplasts and Photosystem II particles from a barley mutant lacking chlorophyll b. - BBA-Bioenergetics 932: 97-106, 1988.

Reinbothe C., Bartsch S., Eggink L.L. et al.: A role for chlorophyllide oxygenase in the regulated import and stabilization of light-harvesting chlorophyll proteins. - P. Natl. Acad. Sci. USA 103: 4777-4782, 2006.

Rühle W., Reiländer H., Otto K.D., Wild A.: Chlorophyllprotein-complexes of thylakoids of wild type and chlorophyll $b$ mutants of Arabidopsis thaliana. - Photosynth. Res. 4: 301-305, 1983.

Sato Y., Morita R., Katsuma S. et al.: Two short-chain dehydrogenase/reductases, NON-YELLOW COLORING 1 and NYC1-LIKE, are required for chlorophyll $b$ and lightharvesting complex II degradation during senescence in rice.Plant J. 57: 120-131, 2009.

Schmitz A.J., Glynn J.M., Olson B.J.S.C. et al.: Arabidopsis FtsZ2-1 and FtsZ2-2 are functionally redundant, but Ftszbased plastid division is not essential for chloroplast partitioning or plant growth and development. - Mol. Plant 2: 1211-1222, 2009.

Seo T.S., Bai X., Ruparel H. et al:: Photocleavable fluorescent nucleotides for DNA sequencing on a chip constructed by site-specific coupling chemistry. - P. Natl. Acad. Sci. USA 101: 5488-5493, 2004.

Shi L.X., Hall M., Funk C., Schröder W.P.: Photosystem II, a growing complex: Updates on newly discovered components and low molecular mass proteins. - BBA-Bioenergetics 1817: 13-25, 2012.

Shimoda Y., Ito H., Tanaka A.: Conversion of chlorophyll $b$ to chlorophyll $a$ precedes magnesium dechelation for protection against necrosis in Arabidopsis. - Plant J. 72: 501-511, 2012.

Spurr A.R.: A low-viscosity epoxy resin embedding medium for electron microscopy. - J. Ultrastruct. Res. 26: 31-43, 1969.

Tanaka A., Tanaka R.: Chlorophyll metabolism. - Curr. Opin. Plant Biol. 9: 248-255, 2006.

Tanaka A., Tanaka R.: The biochemistry, physiology, and evolution of the chlorophyll cycle. - Adv. Bot. Res. 90: 183-
$212,2019$.

Terao T., Sonoike K., Yamazaki J. et al.: Stoichiometries of photosystem I and photosystem II in rice mutants differently deficient in chlorophyll b. - Plant Cell Physiol. 37: 299-306, 1996.

Terao T., Yamashita A., Katoh S.: Chlorophyll b-deficient mutants of rice: I. Absorption and fluorescence spectra and chlorophyll $a / b$ ratios. - Plant Cell Physiol. 26: 1361-1367, 1985a.

Terao T., Yamashita A., Katoh S.: Chlorophyll $b$-deficient mutants of rice: II. Antenna chlorophyll $a / b$-proteins of photosystem I and II. - Plant Cell Physiol. 26: 1369-1377, 1985 b.

Thornber J.P., Highkin H.R.: Composition of the photosynthetic apparatus of normal barley leaves and a mutant lacking chlorophyll $b$. - Eur. J. Biochem. 41: 109-116, 1974.

Wang F., Wang G., Li X. et al.: Heredity, physiology and mapping of a chlorophyll content gene of rice (Oryza sativa L.). J. Plant Physiol. 165: 324-330, 2008.

Wang L., Yue C., Cao H. et al.: Biochemical and transcriptome analyses of a novel chlorophyll-deficient chlorina tea plant cultivar. - BMC Plant Biol. 14: 352, 2014.

Wang Z., Gerstein M., Snyder M.: RNA-Seq: a revolutionary tool for transcriptomics. - Nat. Rev. Genet. 10: 57, 2009.

Yang C.M., Chang K.W., Yin M.H. et al.: Method for the determination of the chlorophylls and their derivatives. Taiwania 43: 116-122, 1998.

Yang C.M., Chen H.Y.: Grana stacking is normal in a chlorophylldeficient LT8 mutant of rice. - Bot. Bull. Acad. Sin. 37: 31-34, 1996.

Yang C.M., Osterman J.C., Markwell J.: Temperature sensitivity as a general phenomenon in a collection of chlorophylldeficient mutants of sweetclover (Melilotus alba). - Biochem. Genet. 28: 31-40, 1990.

Yang H.Y., Xia X.W., Fang W. et al.: Identification of genes involved in spontaneous leaf color variation in Pseudosasa japonica. - Genet. Mol. Res. 14: 11827-11840, 2015a.

Yang Y., Chen X., Xu B. et al:: Phenotype and transcriptome analysis reveals chloroplast development and pigment biosynthesis together influenced the leaf color formation in mutants of Anthurium andraeanum 'Sonate.' - Front. Plant Sci. 6: 139, 2015 b.

Yang Y., Xu J., Huang L. et al.: PGL, encoding chlorophyllide $a$ oxygenase 1, impacts leaf senescence and indirectly affects grain yield and quality in rice. - J. Exp. Bot. 67: 1297-1310, 2015c.

Zhang H., Zhang D., Han S. et al.: Identification and gene mapping of a soybean chlorophyll-deficient mutant. - Plant Breeding 130: 133-138, 2011.

Zhao X., Chen T., Feng B. et al.: Non-photochemical quenching plays a key role in light acclimation of rice plants differing in leaf color. - Front Plant Sci. 7: 1968, 2017.

Zhu H., Zhou Y.Y., Zhai H. et al.: Transcriptome profiling reveals insights into the molecular mechanism of drought tolerance in sweetpotato. - J. Integr. Agr. 18: 9-23, 2019.

(C) The authors. This is an open access article distributed under the terms of the Creative Commons BY-NC-ND Licence. 